\title{
КРАЕВЫЕ ЗАДАЧИ ДЛЯ ОБОБЩЕННОГО МОДИФИЦИРОВАННОГО УРАВНЕНИЯ ВЛАГОПЕРЕНОСА И РАЗНОСТНЫЕ МЕТОДЫ ИХ ЧИСЛЕННОЙ РЕАЛИЗАЦИИ
}

\author{
М. Х. Бештоков \\ (Статъя представлена членом редакционной коллегии А. П. Солдатовым) \\ Институт прикладной математики и автоматизации КБНЦ РАН, \\ г. Нальчик, 360000, Россия \\ E-mail:beshtokov-murat@yandex.ru
}

\begin{abstract}
Аннотация. Рассмотрены начально-краевые задачи с условиями первого и третьего рода для обобщенного модифицированного уравнения влагопереноса с дробной по времени производной. На равномерной сетке построены разностные схемы, аппроксимирующие эти задачи. Для решения этих задач в предположение существования регулярного решения получены априорные оценки в дифференциальной и разностной формах. Из этих оценок следуют единственность и непрерывная зависимость решения от входных данных задачи, а также сходимость со скоростью $O\left(h^{2}+\tau^{2}\right)$.

Ключевые слова: Краевые задачи, априорная оценка, модифицированного уравнение влагопереноса, дифференциальное уравнение дробного порядка, дробная производная Капуто.

Для цитирования: Бештоков М. Х. 2020. Краевые задачи для обобщенного модифицированного уравнения влагопереноса и разностные методы их численной реализации. Прикладная математика \& Физика, $52(2)$ : 128-138. DOI 10.18413/2687-0959-2020-52-2-128-138.

\section{BOUNDARY VALUE PROBLEMS FOR THE GENERALIZED MODIFIED MOISTURE TRANSFER EQUATION AND DIFFERENCE METHODS FOR THEIR NUMERICAL IMPLEMENTATION}

\author{
M. KH. Beshtokov \\ (Article submitted by a member of the editorial board A. P. Soldatov) \\ Institute of Applied Mathematics and Automation KBSC RAS, \\ Nalchik, 360000, Russia \\ E-mail: beshtokov-murat@yandex.ru \\ Received May 28, 2020
}

\begin{abstract}
Initial boundary value problems with conditions of the first and third kind for a generalized modified moisture transfer equation with a time-fractional derivative are considered. Difference schemes approximating these problems are constructed on a uniform grid. To solve these problems, assuming the existence of a regular solution, a priori estimates in differential and difference forms are obtained. From these estimates follow the uniqueness and continuous dependence of the solution on the input data of the problem, as well as convergence with the speed $O\left(h^{2}+\tau^{2}\right)$.

Key words: Boundary Value Problems, a Priori Estimation, Modified Moisture Transfer Equation, Fractional Order Differential Equation, Caputo Fractional Derivative.

For citation: Beshtokov M. KH. 2020. Boundary value problems for the generalized modified moisture transfer equation and difference methods for their numerical implementation. Applied Mathematics \& Physics, 52(2): 128-138 (in Russian).

DOI 10.18413/2687-0959-2020-52-2-128-138.
\end{abstract}

1. Введение. Псевдопараболическими уравнениями в математической литературе последнего времени называют уравнения вида

$$
u_{t}-A u_{t}-B u=f(x, t)
$$

где А и В - операторы второго или более высокого порядка по пространственным переменным [Свешников, Альшин, Корпусов, Плетнер, 2007]. Вопросы, связанные с влагопереносом в почвогрунтах, приводят к псевдопараболическим уравнениям [Чудновский, 1976, с.137]. 
Локальные и нелокальные начально-краевые задачи для такого вида уравнений, как линейных, так и нелинейных, в цилиндрической области $Q=G \times(0, T), G \subset R^{n}$, изучены достаточно хорошо (см., например, [Турбин, 2013; Шергин, Пятков, 2014; Юлдашев, 2016; Юлдашев, 2017; Lyubanova, 2017]).

Многие ученые стали в последнее время изучать уравнения, содержащие дробные производные по временной и пространственным переменным, в связи с тем, что в рамках классической теории дифференциальных уравнений целочисленных порядков многие процессы и явления окружающей среды не поддаются описанию, так как имеют свойство нелокальности и нелинейности как по пространству, так и по времени. Такие явления и процессы обычно описывают с помощью теории дробного исчисления и встречаются они в механике, физике при описании сложных систем различной природы [Gao, Sun, Sun, 2015; Cui, 2013; Gao, Sun, Zhang, 2014; Pang, Sun, 2012; Calcagni, 2012].

Работы [Pimenov, Hendy, 2016a; Pimenov, Hendy, 2016б; Pimenov, 2018] посвящены исследованию различных краевых задач для дифференциальных уравнений в частных производных дробного порядка с эффектом запаздывания по времени. В физическом аспекте понятия память, последействие, запаздывание, наследственность считаются очень близкими.

В настоящей же работе в случае операторов А и В второго порядка для модифицированного уравнения влагопереноса с переменными коэффициентами и дробной по времени производной в смысле Капуто, будут исследоваться первая и третья краевые задачи.

Приближенным методам решения краевых задач для различных уравнений дробного порядка посвящены работы автора [Бештоков, 2018а; Бештоков, 2018б; Бештоков, 2019; Бештоков, Водахова, 2019].

2. Постановка первой краевой задачи. В прямоугольнике $\bar{Q}_{T}=\{(x, t): 0 \leq x \leq l, 0 \leq t \leq T\}$ рассмотрим первую краевую задачу для обобщенного уравнения влагопереноса

$$
\begin{gathered}
\partial_{0 t}^{\alpha} u=\frac{\partial}{\partial x}\left(k(x, t) \frac{\partial u}{\partial x}\right)+\partial_{0 t}^{\alpha} \frac{\partial}{\partial x}\left(\eta(x) \frac{\partial u}{\partial x}\right)+r(x, t) \frac{\partial u}{\partial x}-q(x, t) u+f(x, t), \\
0<x<l, 0<t \leq T, \\
u(0, t)=u(l, t)=0,0 \leq t \leq T, \\
u(x, 0)=u_{0}(x), 0 \leq x \leq l,
\end{gathered}
$$

где

$$
\begin{gathered}
0<c_{0} \leq k(x, t), \eta(x), q(x, t), r_{x}(x, t) \leq c_{1}, \quad|r(x, t)|,\left|k_{x}(x, t)\right| \leq c_{2}, \\
k(x, t) \in C^{1,0}\left(Q_{T}\right), \eta(x) \in C^{1}[0, l], r(x, t), q(x, t), f(x, t) \in C\left(Q_{T}\right), \\
u(x, t) \in C^{2,0}\left(Q_{T}\right) \cap C^{1,0}\left(\bar{Q}_{T}\right), \partial_{0 t}^{\alpha} u(x, t) \in C\left(Q_{T}\right),
\end{gathered}
$$

$\partial_{0 t}^{\alpha} u=\frac{1}{\Gamma(1-\alpha)} \int_{0}^{t} \frac{u_{\tau}(x, \tau)}{(t-\tau)^{\alpha}} d \tau,-$ дробная производная Капуто порядка $\alpha, 0<\alpha<1, c_{i}, i=0,1,2=$ const $>0$.

Далее предполагается, что дифференциальная задача (1)-(3) имеет единственное решение, обладающее нужными по ходу изложения производными.

В работе будем использовать обозначения $M_{i}=$ const $>0, i=1,2, \ldots$, которые зависят только от входных данных рассматриваемой задачи.

\section{3. Априорная оценка в дифференциальной форме.}

Теорема 1. Пусть условия (4) выполнены, тогда для решения задачи (1)-(3) справедлива оцен$\kappa a$

$$
\|u\|_{W_{2}^{1}(0, l)}^{2}+D_{0 t}^{-\alpha}\|u\|_{W_{2}^{1}(0, l)}^{2} \leq M\left(D_{0 t}^{-\alpha}\|f\|_{0}^{2}+\left\|u_{0}(x)\right\|_{W_{2}^{1}(0, l)}^{2}\right)
$$

где $M=$ const $>0$, зависит только от входных данных задачи (1)-(3), $D_{0 t}^{-\alpha} u=\frac{1}{\Gamma(\alpha)} \int_{0}^{t} \frac{u d \tau}{(t-\tau)^{1-\alpha}}-$ дробный интеграл Римана - Лиувилля порядка $\alpha, 0<\alpha<1,\|u\|_{W_{2}^{1}(0, l)}^{2}=\|u\|_{0}^{2}+\left\|u_{x}\right\|_{0}^{2}$.

Доказательство. Получим априорную оценку решения задачи (1)-(3) в дифференциальной форме. Для этого умножим уравнение (1) скалярно на u:

$$
\left(\partial_{0 t}^{\alpha} u, u\right)=\left(\left(k u_{x}\right)_{x}, u\right)+\left(\partial_{0 t}^{\alpha}\left(\eta u_{x}\right)_{x}, u\right)+\left(r u_{x}, u\right)-(q u, u)+(f, u),
$$

где $(a, b)=\int_{0}^{l} a b d x,(a, a)=\|a\|_{0}^{2},-$ скалярное произведение и норма, где $a, b-$ заданные на $[0, l]$ функции. 
Пользуясь леммой [Алиханов, 2010], получим

$$
\begin{gathered}
\left(\partial_{0 t}^{\alpha} u, u\right) \geq \frac{1}{2}\left(1, \partial_{0 t}^{\alpha} u^{2}\right)=\frac{1}{2} \partial_{0 t}^{\alpha}\|u\|_{0}^{2}, \\
\left(\left(k u_{x}\right)_{x}, u\right)=\int_{0}^{l} u\left(k u_{x}\right)_{x} d x=\left.u k u_{x}\right|_{0} ^{l}-\int_{0}^{l} k u_{x}^{2} d x, \\
\left(\partial_{0 t}^{\alpha}\left(\eta u_{x}\right)_{x}, u\right)=\int_{0}^{l} u \partial_{0 t}^{\alpha}\left(\eta u_{x}\right)_{x} d x=\left.u \partial_{0 t}^{\alpha}\left(\eta u_{x}\right)\right|_{0} ^{l}-\int_{0}^{l} \eta(x) u_{x} \partial_{0 t}^{\alpha} u_{x} d x \leq \\
\leq\left. u \partial_{0 t}^{\alpha}\left(\eta u_{x}\right)\right|_{0} ^{l}-\frac{1}{2} \int_{0}^{l} \eta \partial_{0 t}^{\alpha}\left(u_{x}\right)^{2} d x, \\
\left(r u_{x}, u\right)=\int_{0}^{l} r u_{x} u d x=\left.\frac{1}{2} r u^{2}\right|_{0} ^{l}-\frac{1}{2} \int_{0}^{l} r_{x} u^{2} d x \leq\left.\frac{1}{2} r u^{2}\right|_{0} ^{l}-\frac{c_{0}}{2}\|u\|_{0}^{2}, \\
(q u, u)=\int_{0}^{l} q u^{2} d x \geq c_{0}\|u\|_{0}^{2}, \\
(f, u)=\int_{0}^{l} f u d x \leq \varepsilon\|u\|_{0}^{2}+M_{1}(\varepsilon)\|f\|_{0}^{2} .
\end{gathered}
$$

Принимая во внимание преобразования (6)-(11), из (5) находим

$$
\begin{gathered}
\partial_{0 t}^{\alpha}\|u\|_{0}^{2}+\frac{1}{2} \int_{0}^{l} \eta \partial_{0 t}^{\alpha}\left(u_{x}\right)^{2} d x+2 c_{0}\left\|u_{x}\right\|_{0}^{2}+3 c_{0}\|u\|_{0}^{2} \leq \\
\leq\left. 2 u\left(k u_{x}+\partial_{0 t}^{\alpha}\left(\eta u_{x}\right)\right)\right|_{0} ^{l}+\left.r u^{2}\right|_{0} ^{l}+2 \varepsilon\|u\|_{0}^{2}+M_{1}(\varepsilon)\|f\|_{0}^{2} .
\end{gathered}
$$

Выбирая $\varepsilon=c_{0}$, из (12) с учетом (2) получаем

$$
\partial_{0 t}^{\alpha}\|u\|_{0}^{2}+\int_{0}^{l} \eta \partial_{0 t}^{\alpha}\left(u_{x}\right)^{2} d x+\|u\|_{0}^{2}+\left\|u_{x}\right\|_{0}^{2} \leq M_{2}\|f\|_{0}^{2}
$$

Применяя к обеим частям (13) оператор дробного интегрирования $D_{0 t}^{-\alpha}$, из (13) находим

$$
\|u\|_{W_{2}^{1}(0, l)}^{2}+D_{0 t}^{-\alpha}\|u\|_{W_{2}^{1}(0, l)}^{2} \leq M\left(D_{0 t}^{-\alpha}\|f\|_{0}^{2}+\left\|u_{0}(x)\right\|_{W_{2}^{1}(0, l)}^{2}\right)
$$

где $M=$ const $>0$, зависит только от входных данных задачи (1)-(3).

Из (14) следуют единственность и непрерывная зависимость решения от входных данных задачи (1) $-(3)$.

4. Устойчивость и сходимость разностной схемы. Решим задачу (1)-(3) с помощью метода конечных разностей. Для этого дифференциальной задаче (1)-(3) поставим в соответствие на равномерной сетке $\bar{\omega}_{h \tau}$ разностную схему со вторым порядком точности по $h$ и $\tau$ :

$$
\begin{gathered}
\Delta_{0 t_{j+\sigma}}^{\alpha} y=\varkappa_{i}^{j}\left(a_{i}^{j} y_{\bar{x}}^{(\sigma)}\right)_{x, i}+\Delta_{0 t_{j+\sigma}}^{\alpha}\left(\gamma_{i} y_{\bar{x}}\right)_{x, i}+b_{i}^{-j} a_{i}^{j} y_{\bar{x}, i}+b_{i}^{+j} a_{i+1}^{j} y_{x, i}^{(\sigma)}-d_{i}^{j} y_{i}^{(\sigma)}+\varphi_{i}^{j},(x, t) \in \omega_{h, \tau} \\
y_{0}^{(\sigma)}=y_{N}^{(\sigma)}=0 \\
y(x, 0)=u_{0}(x)
\end{gathered}
$$

где $\Delta_{0 t_{j+\sigma}}^{\alpha} y=\frac{\tau^{1-\alpha}}{\Gamma(2-\alpha)} \sum_{s=0}^{j} c_{j-s}^{(\alpha, \sigma)} y_{t}^{s}-$ дискретный аналог дробной производной Капуто порядка $\alpha, 0<$ $\alpha<1$ [Alikhanov, 2015].

$$
\begin{gathered}
a_{0}^{(\alpha, \sigma)}=\sigma^{1-\alpha}, a_{l}^{(\alpha, \sigma)}=(l+\sigma)^{1-\alpha}-(l-1+\sigma)^{1-\alpha}, l \geq 1, \\
b_{l}^{(\alpha, \sigma)}=\frac{1}{2-\alpha}\left[(l+\sigma)^{2-\alpha}-(l-1+\sigma)^{2-\alpha}\right]-\frac{1}{2}\left[(l+\sigma)^{1-\alpha}+(l-1+\sigma)^{1-\alpha}\right], l \geq 1, \\
\text { при } j=0, \quad c_{0}^{(\alpha, \sigma)}=a_{0}^{(\alpha, \sigma)} ;
\end{gathered}
$$




$$
\begin{aligned}
& \text { при } j>0, \quad c_{s}^{(\alpha, \sigma)}=\left\{\begin{array}{l}
a_{0}^{(\alpha, \sigma)}+b_{1}^{(\alpha, \sigma)}, s=0, \\
a_{s}^{(\alpha, \sigma)}+b_{s+1}^{(\alpha, \sigma)}-b_{s}^{(\alpha, \sigma)}, 1 \leq s \leq j-1, \\
a_{j}^{(\alpha, \sigma)}-b_{j}^{(\alpha, \sigma)}, s=j,
\end{array}\right. \\
& a_{i}^{j}=k\left(x_{i-0.5}, t^{j+\sigma}\right), \gamma_{i}=\eta\left(x_{i-0.5}\right), b_{i}^{j}=\frac{r\left(x, t^{j+\sigma}\right)}{k\left(x_{i}, t^{j+\sigma}\right)}, \varphi_{i}^{j}=f\left(x_{i}, t^{j+\sigma}\right), \sigma=1-\frac{\alpha}{2}, \\
& c_{s}^{(\alpha, \sigma)}>\frac{1-\alpha}{2}(s+\sigma)^{-\alpha}>0, y^{(\sigma)}=\sigma y^{j+1}+(1-\sigma) y^{j}, d_{i}^{j}=d\left(x_{i}, t^{j+\sigma}\right) .
\end{aligned}
$$

Теорема 2. Пусть условия (4) выполнены, тогда существует такое $\tau_{0}$, что если $\tau \leq \tau_{0}$, то для решения разностной задачи (15)-(17) справедлива оченка

$$
\left\|y^{j+1}\right\|_{W_{2}^{1}(0, l)}^{2} \leq M\left(\left\|y^{0}\right\|_{W_{2}^{1}(0, l)}^{2}+\max _{0 \leq j^{\prime} \leq j}\left\|\varphi^{j^{\prime}}\right\|_{0}^{2}\right) .
$$

где $M=$ const $>0$, не зависящая от $h u \tau$.

Доказательство. Найдем методом энергетических неравенств априорную оценку в разностной форме, для этого введем скалярные произведения и норму в следующем виде:

$$
(u, v)=\sum_{i=1}^{N-1} u_{i} v_{i} h, \quad(u, v]=\sum_{i=1}^{N} u_{i} v_{i} h,(u, u)=\left(1, u^{2}\right)=\|u\|_{0}^{2} .
$$

Умножим теперь (15) скалярно на $y^{(\sigma)}$ :

$$
\begin{aligned}
\left(\Delta_{0 t_{j+\sigma}}^{\alpha} y, y^{(\sigma)}\right) & =\left(\varkappa\left(a y_{\bar{x}}^{(\sigma)}\right)_{x}, y^{(\sigma)}\right)+\left(\Delta_{0 t_{j+\sigma}}^{\alpha}\left(\gamma_{i} y_{\bar{x}}\right)_{x}, y^{(\sigma)}\right)+\left(b^{-} a y_{\bar{x}}^{(\sigma)}, y^{(\sigma)}\right)+ \\
& +\left(b^{+} a^{(+1)} y_{x}^{(\sigma)}, y^{(\sigma)}\right)-\left(d y^{(\sigma)}, y^{(\sigma)}\right)+\left(\varphi, y^{(\sigma)}\right) .
\end{aligned}
$$

Преобразуем суммы, входящие в тождество (18), с учетом (16) и леммы [Alikhanov, 2015]

$$
\begin{gathered}
\left(\Delta_{0 t_{j+\sigma}}^{\alpha} y, y^{(\sigma)}\right) \geq \frac{1}{2}\left(1, \Delta_{0 t_{j+\sigma}}^{\alpha}\left(y^{2}\right)\right) \\
\left(\varkappa\left(a y_{\bar{x}}^{(\sigma)}\right)_{x}, y^{(\sigma)}\right)=\left.\varkappa a y_{\bar{x}}^{(\sigma)} y^{(\sigma)}\right|_{0} ^{N}-\left(a y_{\bar{x}}^{(\sigma)},\left(\varkappa y^{(\sigma)}\right)_{\bar{x}}\right]=-\left(a \varkappa_{\bar{x}}, y_{\bar{x}}^{(\sigma)} y^{(\sigma)}\right]-\left(a \varkappa^{(-1)},\left(y_{\bar{x}}^{(\sigma)}\right)^{2}\right] \leq \\
\leq-\left(a \varkappa_{\bar{x}}, y_{\bar{x}}^{(\sigma)} y^{(\sigma)}\right]-\frac{1}{\left(1+h M_{1}\right)}\left(a \varkappa,\left(y_{\bar{x}}^{(\sigma)}\right)^{2}\right] ; \\
\left(\Delta_{0 t_{j+\sigma}}^{\alpha}\left(\gamma y_{\bar{x}}\right)_{x}, y^{(\sigma)}\right)=\left.y^{(\sigma)} \Delta_{0 t_{j+\sigma}}^{\alpha}\left(\gamma y_{\bar{x}}\right)\right|_{0} ^{N}-\left(\gamma, y_{\bar{x}}^{(\sigma)} \Delta_{0 t_{j+\sigma}}^{\alpha}\left(y_{\bar{x}}\right)\right] \leq \\
\left.\leq-\left(\frac{\gamma_{i}}{2}, \Delta_{0 t_{j+\sigma}}^{\alpha}\left(y_{\bar{x}}\right)^{2}\right] \leq-\frac{c_{0}}{2} \Delta_{0 t_{j+\sigma}}^{\alpha} \| y_{\bar{x}}\right]_{0}^{2} \\
\left(d y^{(\sigma)}, y^{(\sigma)}\right) \geq c_{0}\left\|y^{(\sigma)}\right\|_{0}^{2} \\
\left(\varphi, y^{(\sigma)}\right) \leq \varepsilon\left\|y^{(\sigma)}\right\|_{0}^{2}+\frac{1}{4 \varepsilon}\|\varphi\|_{0}^{2} .
\end{gathered}
$$

Принимая во внимание преобразования (19)-(23), из (18) находим

$$
\begin{gathered}
\left.\left(\frac{1}{2}, \Delta_{0 t_{j+\sigma}}^{\alpha}\left(y^{2}\right)\right)+\frac{1}{\left(1+h M_{1}\right)}\left(a \varkappa,\left(y_{\bar{x}}^{(\sigma)}\right)^{2}\right]+\frac{c_{0}}{2} \Delta_{0 t_{j+\sigma}}^{\alpha} \| y_{\bar{x}}\right]\left.\right|_{0} ^{2} \leq-\left(a \varkappa_{\bar{x}}, y_{\bar{x}}^{(\sigma)} y^{(\sigma)}\right]+\left(b^{-} a y_{\bar{x}}^{(\sigma)}, y^{(\sigma)}\right)+ \\
+\left(b^{+} a^{(+1)} y_{x}^{(\sigma)}, y^{(\sigma)}\right)-c_{0}\left\|y^{(\sigma)}\right\|_{0}^{2}+\varepsilon\left\|y^{(\sigma)}\right\|_{0}^{2}+\frac{1}{4 \varepsilon}\|\varphi\|_{0}^{2}
\end{gathered}
$$

Выбирая $\varepsilon=\frac{c_{0}}{2}$, из $(24)$ находим

$$
\begin{aligned}
\left.\Delta_{0 t_{j+\sigma}}^{\alpha}\|y\|_{0}^{2}+c_{0} \Delta_{0 t_{j+\sigma}}^{\alpha} \| y_{\bar{x}}\right]\left.\right|_{0} ^{2}+ & \left.M_{2} \| y_{\bar{x}}^{(\sigma)}\right]\left.\right|_{0} ^{2}+c_{0}\left\|y^{(\sigma)}\right\|_{0}^{2} \leq-\left(a \varkappa_{\bar{x}}, y_{\bar{x}}^{(\sigma)} y^{(\sigma)}\right]+\left(b^{-} a, y_{\bar{x}}^{(\sigma)} y^{(\sigma)}\right)+ \\
+ & \left(b^{+} a^{(+1)} y_{x}^{(\sigma)}, y^{(\sigma)}\right)+M_{3}\|\varphi\|_{0}^{2} .
\end{aligned}
$$


Преобразуем первое, второе и третье слагаемые в правой части (25). Тогда получим

$$
\left.-\left(a \varkappa_{\bar{x}}, y_{\bar{x}}^{(\sigma)} y^{(\sigma)}\right]+\left(b^{-} a, y_{\bar{x}}^{(\sigma)} y^{(\sigma)}\right)+\left(b^{+} a^{(+1)} y_{x}^{(\sigma)}, y^{(\sigma)}\right) \leq\left. M_{4}\left(\left\|y^{(\sigma)}\right\|_{0}^{2}+\| y_{\bar{x}}^{(\sigma)}\right]\right|_{0} ^{2}\right) .
$$

Учитывая (26), из (25) получаем

$$
\Delta_{0 t_{j+\sigma}}^{\alpha}\|y\|_{W_{2}^{1}(0, l)}^{2}+\left\|y^{\sigma}\right\|_{W_{2}^{1}(0, l)}^{2} \leq M_{5}\left\|y^{\sigma}\right\|_{W_{2}^{1}(0, l)}^{2}+M_{6}\|\varphi\|_{0}^{2},
$$

где $\left.\|y\|_{W_{2}^{1}(0, l)}^{2}=\|y\|_{0}^{2}+\| y_{\bar{x}}\right]\left.\right|_{0} ^{2}$.

Перепишем (27) в другой форме

$$
\Delta_{0 t_{j+\sigma}}^{\alpha}\|y\|_{W_{2}^{1}(0, l)}^{2} \leq M_{7}^{\sigma}\left\|y^{j+1}\right\|_{W_{2}^{1}(0, l)}^{2}+M_{8}^{\sigma}\left\|y^{j}\right\|_{W_{2}^{1}(0, l)}^{2}+M_{9}\|\varphi\|_{0}^{2} .
$$

На основании леммы 3 [Бештоков, 2018] из (28) получаем

$$
\left\|y^{j+1}\right\|_{W_{2}^{1}(0, l)}^{2} \leq M\left(\left\|y^{0}\right\|_{W_{2}^{1}(0, l)}^{2}+\max _{0 \leq j^{\prime} \leq j}\left\|\varphi^{j^{\prime}}\right\|_{0}^{2}\right)
$$

где $M=$ const $>0$, не зависящая от $h$ и $\tau$.

Из (29) следуют единственность и непрерывная зависимость решения от входных данных задачи $(15)-(17)$, а также в силу линейности задачи (1)-(3) сходимость со скоростью $O\left(h^{2}+\tau^{2}\right)$.

5. Постановка третьей краевой задачи и априорная оценка в дифференциальной форме. Рассмотрим третью краевую задачу для уравнения (1)

$$
\left\{\begin{array}{l}
\Pi(0, t)=\beta_{1}(t) u(0, t)-\mu_{1}(t), \\
-\Pi(l, t)=\beta_{2}(t) u(l, t)-\mu_{2}(t)
\end{array}\right.
$$

где

$$
0<c_{0} \leq k, \eta \leq c_{1},\left|\beta_{1}, \beta_{2}, r, q, r_{x}, k_{x}\right| \leq c_{2}, \Pi(x, t)=k(x, t) u_{x}+\partial_{0 t}^{\alpha}\left(\eta u_{x}\right) .
$$

Теорема 3. Пусть условия (4), (31) выполнены, тогда для решения задачи (1), (30), (3) справедлива оценка

$$
\|u\|_{W_{2}^{1}(0, l)}^{2}+D_{0 t}^{-\alpha}\left\|u_{x}\right\|_{0}^{2} \leq M\left(D_{0 t}^{-\alpha}\left(\|f\|_{0}^{2}+\mu_{1}^{2}(t)+\mu_{2}^{2}(t)\right)+\left\|u_{0}(x)\right\|_{W_{2}^{1}(0, l)}^{2}\right),
$$

где $M=$ const > 0, зависит только от входных данных задачи (1),(30),(3).

Доказательство. Умножим уравнение (1) скалярно на u:

$$
\left(\partial_{0 t}^{\alpha} u, u\right)=\left(\left(k u_{x}\right)_{x}, u\right)+\left(\partial_{0 t}^{\alpha}\left(\eta u_{x}\right)_{x}, u\right)+\left(r u_{x}, u\right)-(q u, u)+(f, u) .
$$

Преобразуем третье и четвертое слагаемые в правой части (32)

$$
\begin{gathered}
\left(r u_{x}, u\right)=\int_{0}^{l} r u u_{x} d x \leq \frac{c_{2}}{2} \int_{0}^{l} u^{2} d x+\frac{c_{2}}{2} \int_{0}^{l} u_{x}^{2} d x \leq \frac{c_{2}}{2}\left(\|u\|_{0}^{2}+\left\|u_{x}\right\|_{0}^{2}\right) . \\
-(q u, u)=-\int_{0}^{l} q u^{2} d x \leq c_{2}\|u\|_{0}^{2} .
\end{gathered}
$$

С учетом преобразований (6)-(8),(11),(33),(34) из (32) находим

$$
\frac{1}{2} \partial_{0 t}^{\alpha}\|u\|_{0}^{2}+\frac{1}{2} \int_{0}^{l} \eta \partial_{0 t}^{\alpha}\left(u_{x}\right)^{2} d x+c_{0}\left\|u_{x}\right\|_{0}^{2} \leq\left. u \Pi(x, t)\right|_{0} ^{l}+M_{1}\left(\|u\|_{0}^{2}+\left\|u_{x}\right\|_{0}^{2}\right)+M_{2}\|f\|_{0}^{2} .
$$

Оценим первое слагаемое в правой части (35)

$$
\begin{gathered}
\left.u \Pi(x, t)\right|_{0} ^{l}=\Pi(l, t) u(l, t)-\Pi(0, t) u(0, t)=u(l, t)\left(\mu_{2}(t)-\beta_{2}(t) u(l, t)\right)+ \\
+u(0, t)\left(\mu_{1}(t)-\beta_{1}(t) u(0, t)\right)=-\beta_{2}(t) u^{2}(l, t)+\mu_{2}(t) u(l, t)-\beta_{1}(t) u^{2}(0, t)+\mu_{1}(t) u(0, t) \leq \\
\leq M_{3}\left(u^{2}(0, t)+u^{2}(l, t)\right)+\frac{1}{2}\left(\mu_{1}^{2}(t)+\mu_{2}^{2}(t)\right) \leq M_{4}\left(\|u\|_{0}^{2}+\left\|u_{x}\right\|_{0}^{2}\right)+\frac{1}{2}\left(\mu_{1}^{2}(t)+\mu_{2}^{2}(t)\right) .
\end{gathered}
$$


Учитывая (36), из (35) получим

$$
\frac{1}{2} \partial_{0 t}^{\alpha}\|u\|_{0}^{2}+\frac{1}{2} \int_{0}^{l} \eta \partial_{0 t}^{\alpha}\left(u_{x}\right)^{2} d x+\left\|u_{x}\right\|_{0}^{2} \leq M_{5}\|u\|_{W_{2}^{1}(0, l)}^{2}+M_{6}\left(\|f\|_{0}^{2}+\mu_{1}^{2}(t)+\mu_{2}^{2}(t)\right) .
$$

Применяя к $(37)$ оператор дробного интегрирования $D_{0 t}^{-\alpha}$, получаем

$$
\begin{gathered}
\|u\|_{W_{2}^{1}(0, l)}^{2}+D_{0 t}^{-\alpha}\left\|u_{x}\right\|_{0}^{2} \leq M_{5} D_{0 t}^{-\alpha}\|u\|_{W_{2}^{1}(0, l)}^{2}+ \\
+M_{7}\left(D_{0 t}^{-\alpha}\left(\|f\|_{0}^{2}+\mu_{1}^{2}(t)+\mu_{2}^{2}(t)\right)+\left\|u_{0}(x)\right\|_{W_{2}^{1}(0, l)}^{2}\right) .
\end{gathered}
$$

На основании леммы [Алиханов, 2010] оценим первое слагаемое в правой части (38).

Пусть $y(t)=D_{0 t}^{-\alpha}\|u\|_{W_{2}^{1}(0, l)}^{2}, \partial_{0 t}^{\alpha} y(t)=\|u(x, t)\|_{W_{2}^{1}(0, l)}^{2}$, тогда получаем

$$
D_{0 t}^{-\alpha}\|u\|_{W_{2}^{1}(0, l)}^{2} \leq M_{8}\left(D_{0 t}^{-2 \alpha}\left(\|f\|_{0}^{2}+\mu_{1}^{2}(t)+\mu_{2}^{2}(t)\right)+\left\|u_{0}(x)\right\|_{W_{2}^{1}(0, l)}^{2}\right) .
$$

В силу того, что для любой неотрицательной функции $g(t)$, интегрируемой на $[0, T]$, справедливо неравенство

$$
D_{0 t}^{-2 \alpha} g(t) \leq \frac{t^{\alpha} \Gamma(\alpha)}{\Gamma(2 \alpha)} D_{0 t}^{-\alpha} g(t)
$$

то из (38) с учетом (39) и (40) находим оценку

$$
\|u\|_{W_{2}^{1}(0, l)}^{2}+D_{0 t}^{-\alpha}\left\|u_{x}\right\|_{0}^{2} \leq M\left(D_{0 t}^{-\alpha}\left(\|f\|_{0}^{2}+\mu_{1}^{2}(t)+\mu_{2}^{2}(t)\right)+\left\|u_{0}(x)\right\|_{W_{2}^{1}(0, l)}^{2}\right)
$$

где $M=$ const $>0$, зависит только от входных данных задачи $(1),(30),(3)$.

Из (41) следуют единственность и непрерывная зависимость решения от входных данных задачи (1), (30), (3).

6. Устойчивость и сходимость разностной схемы. Дифференциальной задаче (1), (30), (3) поставим в соответствие на равномерной сетке $\bar{\omega}_{h \tau}$ разностную схему со вторым порядком точности по $h$ и $\tau$ :

$$
\begin{gathered}
\Delta_{0 t_{j+\sigma}}^{\alpha} y=\varkappa_{i}^{j}\left(a_{i}^{j} y_{\bar{x}}^{(\sigma)}\right)_{x, i}+\Delta_{0 t_{j+\sigma}}^{\alpha}\left(\gamma_{i} y_{\bar{x}}\right)_{x, i}+b_{i}^{-j} a_{i}^{j} y_{\bar{x}, i}^{(\sigma)}+b_{i}^{+j} a_{i+1}^{j} y_{x, i}^{(\sigma)}-d_{i}^{j} y_{i}^{(\sigma)}+\varphi_{i}^{j}, \quad(x, t) \in \omega_{h, \tau} \\
\varkappa_{0} a_{1} y_{x, 0}^{(\sigma)}+\Delta_{0 t_{j+\sigma}}^{\alpha}\left(\gamma_{1} y_{\bar{x}, 0}\right)=\tilde{\beta}_{1} y_{0}^{(\sigma)}+0.5 h \Delta_{0 t_{j+\sigma}}^{\alpha} y_{0}-\tilde{\mu}_{1}, t \in \bar{\omega}_{\tau}, x=0 \\
-\left(\varkappa_{N} a_{N} y_{\bar{x}, N}^{(\sigma)}+\Delta_{0 t_{j+\sigma}}^{\alpha}\left(\gamma_{N} y_{\bar{x}, N}\right)\right)=\tilde{\beta}_{2} y_{N}^{(\sigma)}+0.5 h \Delta_{0 t_{j+\sigma}}^{\alpha} y_{N}-\tilde{\mu}_{2}, t \in \bar{\omega}_{\tau}, x=l \\
y(x, 0)=u_{0}(x), x \in \bar{\omega}_{h}
\end{gathered}
$$

где

$$
\begin{array}{ll}
\tilde{\beta}_{1}\left(t_{j+\sigma}\right)=\beta_{1}\left(t_{j+\sigma}\right)+0.5 h d_{0}^{j}, & \tilde{\beta}_{2}\left(t_{j+\sigma}\right)=\beta_{2}\left(t_{j+\sigma}\right)+0.5 h d_{N}^{j}, \\
\tilde{\mu}_{1}\left(t_{j+\sigma}\right)=\mu_{1}\left(t_{j+\sigma}\right)+0.5 h \varphi_{0}, & \tilde{\mu}_{2}\left(t_{j+\sigma}\right)=\mu_{2}\left(t_{j+\sigma}\right)+0.5 h \varphi_{N},
\end{array}
$$

$\Delta_{0 t_{j+\sigma}}^{\alpha} y=\frac{\tau^{1-\alpha}}{\Gamma(2-\alpha)} \sum_{s=0}^{j} c_{j-s}^{(\alpha, \sigma)} y_{t}^{s}-$ дискретный аналог дробной производной Капуто порядка $\alpha, 0<\alpha<$ 1.

Перепишем (42)-(45) в операторном виде

$$
\left\{\begin{array}{l}
\Delta_{0 t_{j+\sigma}}^{\alpha} y=\bar{\Lambda}\left(t_{j+\sigma}\right) y^{(\sigma)}+\Delta_{0 t_{j+\sigma}}^{\alpha} \bar{\delta}(t) y+\bar{\Phi} \\
y(x, 0)=u_{0}(x), x \in \bar{\omega}_{h}
\end{array}\right.
$$

где

$$
\begin{gathered}
\bar{\Lambda}\left(t_{j+\sigma}\right) y^{(\sigma)}=\left\{\begin{array}{l}
\tilde{\Lambda} y_{i}^{(\sigma)}=\varkappa\left(a y_{\bar{x}}^{(\sigma)}\right)_{x}+b^{-} a y_{\bar{x}}^{(\sigma)}+b^{+} a^{(+1)} y_{x}^{(\sigma)}-d y^{(\sigma)}, i=\overline{1, N-1}, \\
\Lambda^{-} y_{0}^{(\sigma)}=\frac{\varkappa_{0} a_{1} y_{x, 0}^{(\sigma)}-\tilde{\beta}_{1} y_{0}^{(\sigma)}}{0.5 h}, i=0, \\
\Lambda^{+} y_{N}^{(\sigma)}=\frac{-\varkappa_{N} a_{N} y_{\bar{x}, N}^{(\sigma)}-\tilde{\beta}_{2} y_{N}^{(\sigma)}}{0.5 h}, i=N,
\end{array}\right. \\
\bar{\delta} y=\left\{\begin{array}{l}
\delta y_{i}=\left(\gamma_{i} y_{\bar{x}}\right)_{x}, i=\frac{1, N-1,}{1, N}, \quad \bar{\Phi}=\left\{\begin{array}{l}
\varphi=\varphi_{i}, i=\overline{1, N-1} \\
\delta^{-} y_{0}=\frac{2}{h}\left(\gamma_{1} y_{x, 0}\right)_{t}, i=0, i=0, \\
\delta^{+} y_{N}=-\frac{2}{h}\left(\gamma_{N} y_{\bar{x}, N}\right) i=N,
\end{array} \quad i=\frac{2}{h} \tilde{\mu}_{2}, i=N,\right.
\end{array}\right.
\end{gathered}
$$




$$
\varkappa^{*}=\left\{\begin{array}{l}
\varkappa=\frac{1}{1+\frac{0.5 h|r|}{k}}, \\
\varkappa_{0}=\frac{1}{1+\frac{0.5 h\left|r_{0}\right|}{k_{0.5}}}, r_{0} \leq 0, \\
\varkappa_{N}=\frac{1}{1+\frac{0.5 h\left|r_{N}\right|}{k_{N-0.5}}}, r_{N} \geq 0,
\end{array} \quad t^{*}=t^{j+1 / 2} .\right.
$$

Теорема 4. Пусть условия (4), (31) выполнены, тогда существует такое $\tau$, что если $\tau \leq \tau_{0}$ то для решения разностной задачи (42), (45) справедлива оценка

$$
\left|\left[y^{j+1}\right]\right|_{W_{2}^{1}(0, l)}^{2} \leq M\left(\left|\left[y^{0}\right]\right|_{W_{2}^{1}(0, l)}^{2}+\max _{0 \leq j^{\prime} \leq j}\left(\left|\left[\varphi^{j^{\prime}}\right]\right|_{0}^{2}+\mu_{1}^{2}+\mu_{2}^{2}\right)\right)
$$

где $M=$ const $>0$, не зависящая от $h u \tau$.

Доказательство. Умножим (46) теперь скалярно на $y^{(\sigma)}$ :

$$
\left[\Delta_{0 t_{j+\sigma}}^{\alpha} y, y^{(\sigma)}\right]=\left[\bar{\Lambda}\left(t_{j+\sigma}\right) y^{(\sigma)}, y^{(\sigma)}\right]+\left[\Delta_{0 t_{j+\sigma}}^{\alpha} \bar{\delta} y, y^{(\sigma)}\right]+\left[\Phi, y^{(\sigma)}\right]
$$

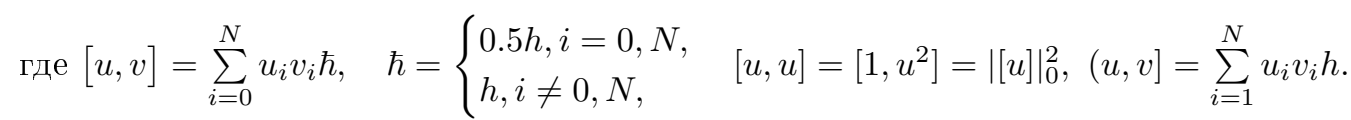

Оценим суммы, входящие в (47)

$$
\begin{gathered}
{\left[\Delta_{0 t_{j+\sigma}}^{\alpha} y, y^{(\sigma)}\right] \geq \frac{1}{2}\left[1, \Delta_{0 t_{j+\sigma}}^{\alpha}\left(y^{2}\right)\right]} \\
{\left[\bar{\Lambda}\left(t_{j+\sigma}\right) y^{(\sigma)}, y^{(\sigma)}\right]=\left(\tilde{\Lambda}\left(t_{j+\sigma}\right) y^{(\sigma)}, y^{(\sigma)}\right)+0.5 h y_{0}^{(\sigma)} \Lambda^{-} y_{0}^{(\sigma)}+0.5 h y_{N}^{(\sigma)} \Lambda^{+} y_{N}^{(\sigma)}=\left(\varkappa\left(a y_{\bar{x}}^{(\sigma)}\right)_{x}, y^{(\sigma)}\right)+} \\
+\left(b^{-} a y_{\bar{x}}^{(\sigma)}, y^{(\sigma)}\right)+\left(b^{+} a^{(+1)} y_{x}^{(\sigma)}, y^{(\sigma)}\right)-\left(d y^{(\sigma)}, y^{(\sigma)}\right)+\varkappa_{0} a_{1} y_{x, 0}^{(\sigma)} y_{0}^{(\sigma)}-\tilde{\beta}_{1}\left(y_{0}^{(\sigma)}\right)^{2}-\varkappa_{N} a_{N} y_{\bar{x}, N}^{(\sigma)} y_{N}^{(\sigma)}- \\
-\tilde{\beta}_{2}\left(y_{N}^{(\sigma)}\right)^{2}=-\left(a y_{\bar{x}}^{(\sigma)},\left(\varkappa y^{(\sigma)}\right)_{\bar{x}}\right]+\left(b^{-} a, y_{\bar{x}}^{(\sigma)} y^{(\sigma)}\right)+\left(b^{+} a^{(+1)}, y_{x}^{(\sigma)} y^{(\sigma)}\right)-\left(d y^{(\sigma)}, y^{(\sigma)}\right)- \\
-\tilde{\beta}_{1}\left(y_{0}^{(\sigma)}\right)^{2}-\tilde{\beta}_{2}\left(y_{N}^{(\sigma)}\right)^{2} .
\end{gathered}
$$

Преобразуем слагаемые в правой части (49)

$$
\begin{gathered}
-\left(a y_{\bar{x}}^{(\sigma)},\left(\varkappa y^{(\sigma)}\right)_{\bar{x}}\right]+\left(b^{-} a, y_{\bar{x}}^{(\sigma)} y^{(\sigma)}\right)+\left(b^{+} a^{(+1)}, y_{x}^{(\sigma)} y^{(\sigma)}\right)=-\left(a \varkappa^{(-1)},\left(y_{\bar{x}}^{(\sigma)}\right)^{2}\right]-\left(a \varkappa_{\bar{x}}, y_{\bar{x}}^{(\sigma)} y^{(\sigma)}\right]+ \\
\left.+\left(b^{-} a, y_{\bar{x}}^{(\sigma)} y^{(\sigma)}\right)+\left(b^{+} a^{(+1)}, y_{x}^{(\sigma)} y^{(\sigma)}\right) \leq-\left(\frac{\varkappa a}{1+h M_{1}},\left(y_{\bar{x}}^{\sigma}\right)^{2}\right]+\left.M_{1}\left(\left|\left[y^{(\sigma)}\right]\right|_{0}^{2}+\| y_{\bar{x}}^{(\sigma)}\right]\right|_{0} ^{2}\right) \\
-\left(d y^{(\sigma)}, y^{(\sigma)}\right)-\widetilde{\beta}_{1}\left(y_{0}^{(\sigma)}\right)^{2}-\widetilde{\beta}_{2}\left(y_{N}^{(\sigma)}\right)^{2}=-\left(d y^{(\sigma)}, y^{(\sigma)}\right)-0.5 h d_{0}\left(y_{0}^{(\sigma)}\right)^{2}-0.5 h d_{N}\left(y_{N}^{(\sigma)}\right)^{2}-\beta_{1}\left(y_{0}^{(\sigma)}\right)^{2}- \\
\left.-\beta_{2}\left(y_{N}^{(\sigma)}\right)^{2}=-\left[d,\left(y^{(\sigma)}\right)^{2}\right]-\beta_{1}\left(y_{0}^{(\sigma)}\right)^{2}-\beta_{2}\left(y_{N}^{(\sigma)}\right)^{2} \leq\left. M_{2}\left(\left|\left[y^{(\sigma)}\right]\right|_{0}^{2}+\| y_{\bar{x}}^{(\sigma)}\right]\right|_{0} ^{2}\right) .
\end{gathered}
$$

Учитывая (50),(51), из (49) находим

$$
\begin{gathered}
\left.\left.\left[\bar{\Lambda}\left(t_{j+\sigma}\right) y^{(\sigma)}, y^{(\sigma)}\right] \leq-M_{3} \| y_{\bar{x}}^{(\sigma)}\right]\left.\right|_{0} ^{2}+\left.M_{4}\left(\left|\left[y^{(\sigma)}\right]\right|_{0}^{2}+\| y_{\bar{x}}^{(\sigma)}\right]\right|_{0} ^{2}\right) . \\
{\left[\Delta_{0 t_{j+\sigma}}^{\alpha} \bar{\delta} y, y^{(\sigma)}\right]=\left(\Delta_{0 t_{j+\sigma}}^{\alpha} \delta y, y^{(\sigma)}\right)+0.5 h y_{0}^{(\sigma)} \Delta_{0 t_{j+\sigma}}^{\alpha} \delta^{-} y_{0}+0.5 h y_{N}^{(\sigma)} \Delta_{0 t_{j+\sigma}}^{\alpha} \delta^{+} y_{N}=} \\
=-\left.\left(y_{\bar{x}}^{(\sigma)}, \Delta_{0 t_{j+\sigma}}^{\alpha}\left(\gamma_{i} y_{\bar{x})}\right] \leq-\left(\frac{\gamma}{2}, \Delta_{0 t_{j+\sigma}}^{\alpha}\left(y_{\bar{x}}\right)^{2}\right] \leq-\frac{c_{0}}{2} \Delta_{0 t_{j+\sigma}}^{\alpha} \| y_{\bar{x}}\right]\right|_{0} ^{2} . \\
{\left[\bar{\Phi}, y^{(\sigma)}\right]=\left(\varphi, y^{(\sigma)}\right)+0.5 h y_{0}^{(\sigma)} \varphi^{-}+0.5 h y_{N}^{(\sigma)} \varphi^{+}=\left[\varphi, y^{(\sigma)}\right]+\mu_{1} y_{0}^{(\sigma)}+\mu_{2} y_{N}^{(\sigma)} \leq} \\
\left.\leq\left. M_{5}\left(\left|\left[y^{(\sigma)}\right]\right|_{0}^{2}+\| y_{\bar{x}}^{(\sigma)}\right]\right|_{0} ^{2}\right)+M_{6}\left(|[\varphi]|_{0}^{2}+\mu_{1}^{j 2}+\mu_{2}^{j 2}\right) .
\end{gathered}
$$

Принимая во внимание преобразования (48)-(54), из (47) находим

$$
\left.\Delta_{0 t_{j+\sigma}}^{\alpha}|[y]|_{W_{2}^{1}(0, l)}^{2}+M_{3} \| y_{\bar{x}}^{(\sigma)}\right]\left.\right|_{0} ^{2} \leq M_{7}\left|\left[y^{(\sigma)}\right]\right|_{W_{2}^{1}(0, l)}^{2}+M_{6}\left(|[\varphi]|_{0}^{2}+\mu_{1}^{2}+\mu_{2}^{2}\right)
$$

где $\left.|[y]|_{W_{2}^{1}(0, l)}^{2}=|[y]|_{0}^{2}+\| y_{\bar{x}}\right]\left.\right|_{0} ^{2}$. 
Из (55) на основании леммы 3 [Бештоков, 2018] находим оценку

$$
\left|\left[y^{j+1}\right]\right|_{W_{2}^{1}(0, l)}^{2} \leq M\left(\left|\left[y^{0}\right]\right|_{W_{2}^{1}(0, l)}^{2}+\max _{0 \leq j^{\prime} \leq j}\left(\left|\left[\varphi^{j^{\prime}}\right]\right|_{0}^{2}+\mu_{1}^{2}+\mu_{2}^{2}\right)\right),
$$

где $M=$ const $>0$, не зависящая от $h$ и $\tau$.

Из (56) следуют единственность и непрерывная зависимость решения от входных данных задачи $(42),(45)$, а также в силу линейности задачи $(1),(30),(3)$ сходимость со скоростью $O\left(h^{2}+\tau^{2}\right)$.

7. Алгоритм приближенного решения разностной задачи, аппроксимирующей третью краевую задачу для обобщенного модифицированного уравнения влагопереноса. Приведем разностную схему (42)-(45) к расчетному виду для приближенного решения. Тогда уравнение (42) приводится к следующему виду

$$
A_{i} y_{i-1}^{j+1}-C_{i} y_{i}^{j+1}+B_{i} y_{i+1}^{j+1}=-F_{i}^{j}, i=\overline{1, N-1},
$$

где

$$
\begin{aligned}
& A_{i}=\tau \sigma \varkappa_{i}^{j} a_{i}^{j}+\gamma_{i} \frac{\tau^{1-\alpha} c_{0}^{(\alpha, \sigma)}}{\Gamma(2-\alpha)}-\tau h \sigma b_{i}^{-j} a_{i}, \quad B_{i}=\tau \sigma \varkappa_{i}^{j} a_{i+1}^{j}+\gamma_{i+1} \frac{\tau^{1-\alpha} c_{0}^{(\alpha, \sigma)}}{\Gamma(2-\alpha)}+\tau h \sigma b_{i}^{+j} a_{i+1}, \\
& C_{i}=A_{i}+B_{i}+h^{2} \frac{\tau^{1-\alpha} c_{0}^{(\alpha, \sigma)}}{\Gamma(2-\alpha)}+\tau \sigma h^{2} d_{i}^{j} \\
& F_{i}^{j}=A A_{i} y_{i-1}^{j}-C C_{i} y_{i}^{j}+B B_{i} y_{i+1}^{j}+h^{2} \tau \varphi_{i}^{j}-h^{2} \frac{\tau^{1-\alpha}}{\Gamma(2-\alpha)} \sum_{s=0}^{j-1} c_{j-s}^{(\alpha, \sigma)}\left(y_{i}^{s+1}-y_{i}^{s}\right)+ \\
& +\frac{\tau^{1-\alpha}}{\Gamma(2-\alpha)} \sum_{s=0}^{j-1} c_{j-s}^{(\alpha, \sigma)}\left(\left(\gamma_{i+1} y_{i+1}\right)^{s+1}-\left(\gamma_{i+1} y_{i+1}\right)^{s}\right)- \\
& -\frac{\tau^{1-\alpha}}{\Gamma(2-\alpha)} \sum_{s=0}^{j-1} c_{j-s}^{(\alpha, \sigma)}\left(\left(\left(\gamma_{i}+\gamma_{i+1}\right) y_{i}\right)^{s+1}-\left(\left(\gamma_{i}+\gamma_{i+1}\right) y_{i}\right)^{s}\right)+ \\
& +\frac{\tau^{1-\alpha}}{\Gamma(2-\alpha)} \sum_{s=0}^{j-1} c_{j-s}^{(\alpha, \sigma)}\left(\left(\gamma_{i} y_{i-1}\right)^{s+1}-\left(\gamma_{i} y_{i-1}\right)^{s}\right), \\
& A A_{i}=\tau(1-\sigma) \varkappa_{i}^{j} a_{i}^{j}-\gamma_{i} \frac{\tau^{1-\alpha} c_{0}^{(\alpha, \sigma)}}{\Gamma(2-\alpha)}-\tau h(1-\sigma) b_{i}^{-j} a_{i}, \\
& B B_{i}=\tau(1-\sigma) \varkappa_{i}^{j} a_{i+1}^{j}-\gamma_{i+1} \frac{\tau^{1-\alpha} c_{0}^{(\alpha, \sigma)}}{\Gamma(2-\alpha)}+\tau h(1-\sigma) b_{i}^{+j} a_{i+1}, \\
& C C_{i}=A A_{i}+B B_{i}-h^{2} \frac{\tau^{1-\alpha} c_{0}^{(\alpha, \sigma)}}{\Gamma(2-\alpha)}+\tau(1-\sigma) h^{2} d_{i}^{j} .
\end{aligned}
$$

Краевое условие (43) принимает вид

$$
y_{0}=\varkappa_{1} y_{1}+\mu_{1}
$$

где

$$
\begin{gathered}
\varkappa_{1}=\frac{\tau \sigma \varkappa_{0} a_{1}+\gamma_{1} \frac{\tau^{1-\alpha} c_{0}^{(\alpha, \sigma)}}{\Gamma(2-\alpha)}}{\tau \sigma \varkappa_{0} a_{1}^{j}+\gamma_{1} \frac{\tau^{1-\alpha} c_{0}^{(\alpha, \sigma)}}{\Gamma(2-\alpha)}+\sigma h \tau \widetilde{\beta}_{1}^{j}+0.5 h^{2} \frac{\tau^{1-\alpha} c_{0}^{(\alpha, \sigma)}}{\Gamma(2-\alpha)}}, \\
\mu_{1}=\left[\widetilde{\mu}_{1} h \tau-(1-\sigma) h \tau \widetilde{\beta}_{1} y_{0}^{j}+\tau(1-\sigma) \varkappa_{0} a_{1}\left(y_{1}^{j}-y_{0}^{j}\right)-\gamma_{1} \frac{\tau^{1-\alpha} c_{0}^{(\alpha, \sigma)}}{\Gamma(2-\alpha)}\left(y_{1}^{j}-y_{0}^{j}\right)+0.5 h^{2} \frac{\tau^{1-\alpha} c_{0}^{(\alpha, \sigma)}}{\Gamma(2-\alpha)} y_{0}-\right. \\
-0.5 h^{2} \frac{\tau^{1-\alpha}}{\Gamma(2-\alpha)} \sum_{s=0}^{j-1} c_{j-s}^{(\alpha, \sigma)}\left(y_{0}^{s+1}-y_{0}^{s}\right)+\frac{\tau^{1-\alpha}}{\Gamma(2-\alpha)} \sum_{s=0}^{j-1} c_{j-s}^{(\alpha, \sigma)}\left(\left(\gamma_{1} y_{1}\right)^{s+1}-\left(\gamma_{1} y_{1}\right)^{s}\right)- \\
\left.-\frac{\tau^{1-\alpha}}{\Gamma(2-\alpha)} \sum_{s=0}^{j-1} c_{j-s}^{(\alpha, \sigma)}\left(\left(\gamma_{1} y_{0}\right)^{s+1}-\left(\gamma_{1} y_{0}\right)^{s}\right)\right] /\left[\tau \sigma \varkappa_{0} a_{1}^{j}+\gamma_{1} \frac{\tau^{1-\alpha} c_{0}^{(\alpha, \sigma)}}{\Gamma(2-\alpha)}+\sigma h \tau \widetilde{\beta}_{1}^{j}+0.5 h^{2} \frac{\tau^{1-\alpha} c_{0}^{(\alpha, \sigma)}}{\Gamma(2-\alpha)}\right] .
\end{gathered}
$$


Краевое условие (44) принимает вид

$$
y_{N}=\varkappa_{2} y_{N-1}+\mu_{2}
$$

где

$$
\begin{gathered}
\varkappa_{2}=\frac{\tau \sigma \varkappa_{N} a_{N}+\gamma_{N} \frac{\tau^{1-\alpha} c_{0}^{(\alpha, \sigma)}}{\Gamma(2-\alpha)}}{\tau \sigma \varkappa_{N} a_{N}^{j}+\gamma_{N} \frac{\tau^{1-\alpha} c_{0}^{(\alpha, \sigma)}}{\Gamma(2-\alpha)}+\sigma h \tau \widetilde{\beta}_{2}^{j}+0.5 h^{2} \frac{\tau^{1-\alpha} c_{0}^{(\alpha, \sigma)}}{\Gamma(2-\alpha)}}, \\
\mu_{2}=\left[\widetilde{\mu}_{2} h \tau-(1-\sigma) h \tau \widetilde{\beta}_{2} y_{N}^{j}-\tau(1-\sigma) \varkappa_{N} a_{N}\left(y_{N}^{j}-y_{N-1}^{j}\right)+\gamma_{N} \frac{\tau^{1-\alpha} c_{0}^{(\alpha, \sigma)}}{\Gamma(2-\alpha)}\left(y_{N}^{j}-y_{N-1}^{j}\right)+\right. \\
+0.5 h^{2} \frac{\tau^{1-\alpha} c_{0}^{(\alpha, \sigma)}}{\Gamma(2-\alpha)} y_{N}-0.5 h^{2} \frac{\tau^{1-\alpha}}{\Gamma(2-\alpha)} \sum_{s=0}^{j-1} c_{j-s}^{(\alpha, \sigma)}\left(y_{N}^{s+1}-y_{N}^{s}\right)- \\
+\frac{\tau^{1-\alpha}}{\Gamma(2-\alpha)} \sum_{s=0}^{j-1} c_{j-s}^{(\alpha, \sigma)}\left(\left(\gamma_{N} y_{N}\right)^{s+1}-\left(\gamma_{N} y_{N}\right)^{s}\right)+ \\
\left.\tau^{1-\alpha} \sum_{s=0}^{j-1} c_{j-s}^{(\alpha, \sigma)}\left(\left(\gamma_{N} y_{N-1}\right)^{s+1}-\left(\gamma_{N} y_{N-1}\right)^{s}\right)\right] /\left[\sigma \varkappa_{N} a_{N}^{j}+\gamma_{N} \frac{\tau^{1-\alpha} c_{0}^{(\alpha, \sigma)}}{\Gamma(2-\alpha)}+\right. \\
\left.+\sigma h \tau \widetilde{\beta}_{2}^{j}+\frac{h^{2}}{2} \frac{\tau^{1-\alpha} c_{0}^{(\alpha, \sigma)}}{\Gamma(2-\alpha)}\right] .
\end{gathered}
$$

Таким образом, с учетом (57)-(59), решение разностной схемы (42)-(45) можно найти методом прогонки.

8. Заключение. В работе исследованы начально-краевые задачи с условиями первого и третьего рода для обобщенного модифицированного уравнения влагопереноса с дробной по времени производной. На равномерной сетке построены разностные схемы, аппроксимирующие эти задачи. Для решения этих задач в предположение существования регулярного решения получены априорные оценки в дифференциальной и разностной формах. Из этих оценок следуют единственность и непрерывная зависимость решения от входных данных задачи, а также сходимость со скоростью $O\left(h^{2}+\tau^{2}\right)$.

\section{Список литературы}

1. Алиханов А. А. 2010. Априорные оценки решений краевых задач для уравнений дробного порядка. Дифференц. уранвения, 46(5): 660-666.

2. Бештоков М. Х. 2018. Локальные и нелокальные краевые задачи для вырождающихся и невырождающихся псевдопараболических уравнений с дробной производной Римана - Лиувилля. Дифференц. уравнения, 54(6): 763-778.

3. Бештоков М. Х. 2018. К краевым задачам для вырождающихся псевдопараболических уравнений с дробной производной Герасимова - Капуто. Известия вузов. Математика, 62(10): 3-16.

4. Бештоков М. Х. 2019. Краевые задачи для нагруженных псевдопараболических уравнений дробного порядка и разностные методы их решения. Известия высших учебных заведений. Математика, Известия вузов. Математика, 63(2): 3-12.

5. Бештоков М. Х., Водахова В. А. 2019. Сеточные методы решения нелокальных краевых задач для уравнения конвекции-диффузии дробного порядка с вырождением. Научные ведомости Белгородского государственного университета. Серия: Математика. Физика, 51(3):347-365.

6. Свешников А. А., Альшин А. Б., Корпусов М. О. Плетнер Ю. Д. 2007. Линейные и нелинейные уравнения соболевского типа. М., Физматлит, 736.

7. Чудновский А. Ф. 1976. Теплофизика почв. М., Наука, 352.

8. Турбин М. В. 2013. Исследование начально-краевой задачи для модели движения жидкости Гершель - Балкли. Вестник Воронежского государственного университета. Серия: Физика. Математика, 2: 246-257.

9. Шергин С. Н., Пятков С. Г. 2014. О некоторых классах обратных задач для псевдопараболических уравнений. Математические заметки СВФУ, 21(2): 106-116 
10. Юлдашев Т. К. 2016. Нелинейное интегро-дифференциальное уравнение псевдопараболического типа с нелокальным интегральным условием. Вестник Волгоградского государственного университета. Серия 1: Математика. Физика, 1(32): 11-23.

11. Юлдашев Т. К. 2017. Нелокальная краевая задача для неоднородного псевдопараболического интегро-дифференциального уравнения с вырожденным ядром. Вестник Волгоградского государственного университета. Серия 1: Математика. Физика, 1(38): 42-54.

12. Alikhanov A. A. 2015. A new difference scheme for the time fractional diffusion equation. Journal of Computational Physics, 280: 424-438.

13. Lyubanova A. Sh. 2017. The inverse problem for the nonlinear pseudoparabolic equation of filtration type, J. Sib. Fed. Univ. Math. Phys., 10(1): 4-15.

14. Gao G. H., Sun H. W., Sun Z. Z. 2015. Stability and convergence of finite difference schemes foraclass of time-fractional sub-diffusion equations based oncertain superconvergence. J. Comput. Phys., 280: 510-528.

15. Cui M. 2013. Convergence analysis of high-order compact alternating direction implicit schemes for the two-dimensional time fractional diffusion equation. Numer. Algorithms, 62: 383-409.

16. Gao G. H., Sun Z. Z., Zhang H. 2014. A new fractional numerical differentiation formula to approximate the Caputo fractional derivative and its applications. J.Comput. Phys., 259: 33--50.

17. Pang H. K., Sun H. W. 2012. Multigrid method for fractional diffusion equations. J. Comput. Phys., 231: 693--703.

18. Calcagni G. 2012. Geometry of Fractional Spaces. Adv. Theor. Math. Phys., 16(2): 549--644.

19. Pimenov V. G., Hendy A. S. 2016. An implicit numerical method for the solution of the fractional advection-diffusion equation with delay. Trudy Inst. Mat. i Mekh. UrO RAN, 22(2): 218-226.

20. Pimenov V. G., Hendy A. S. 2016. Fractional analog of crank-nicholson method for the two sided space fractional partial equation with functional delay. Ural Math. J., 2(1): 48--57.

21. Pimenov V. G. 2018. Numerical methods for fractional advection-diffusion equation with heredity. J. Math. Sci. (N. Y.), 230(5): 737-741.

\section{References}

1. Alihanov A. A. 2010. Apriornye ocenki reshenij kraevyh zadach dlya uravnenij drobnogo poryadka [Priori Estimates for Solutions of Boundary Value Problems for Fractional-Order Equations] Differenc. uravneniya, 46(7): 949--961.

2. Beshtokov M. H. 2018. Lokal'nye i nelokal'nye kraevye zadachi dlya vyrozhdayushchihsya i nevyrozhdayushchihsya psevdoparabolicheskih uravnenij s drobnoj proizvodnoj Rimana-Liuvillya [Local and Nonlocal Boundary Value Problems for Degenerating and Nondegenerating Pseudoparabolic Equations with a Riemann-Liouville Fractional Derivative] Differenc. uravneniya, 54(6): 758-774.

3. Beshtokov M. H. 2018. K kraevym zadacham dlya vyrozhdayushchihsya psevdoparabolicheskih uravnenij s drobnoj proizvodnoj Gerasimova-Kaputo [To Boundary-Value Problems for Degenerating Pseudo- parabolic Equations With Gerasimov-Caputo Fractional Derivative] Russian Mathematics, 62(10): 1-14.

4. Beshtokov M. H. 2019. Kraevye zadachi dlya nagruzhennyh psevdoparabolicheskih uravnenij drobnogo poryadka i raznostnye metody ih resheniya [Boundary-ValueProblems for Loaded Pseudoparabolic Equations of Fractional Order and Difference Methods of Their Solving] Russian Mathematics. 63(2): 1-10.

5. Beshtokov M. H., Vodakhova V. A. 2019. Setevyye metody resheniya nelokal'nykh krayevykh zadach dlya uravneniy konvektsii-diffuzii drobnogo poryadka s vyrozhdeniyem [Grid methods for solving nonlocal boundary value problems for the convection-diffusion equation of fractional order with degeneration]. Belgorod State University Scientific Bulletin. Mathematics. Physics, 51(3): 347-365.

6. Sveshnikov A. A., Alshin A. B., Korpusov M. O. Pletner Yu. D. 2007. Lineynyye i nelineynyye uravneniya sobolevskogo tipa. [Linear and nonlinear Sobolev type equations] M., Fizmatlit, 736. 
7. Chudnovskij A.F. 1976. Teplofizika pochv [Thermophysics of soils] M., Nauka, 352.

8. Turbin M. V. 2013. Issledovaniye nachal'no-krayevoy zadachi dlya modeli dvizheniya zhidkosti Gershel'-Balkli [Investigation of the initial-boundary value problem for the Herschel-Balkley fluid motion model] Bulletin of Voronezh State University. Series: Physics. Mathematics, 2: 246-257.

9. Shergin S. N., Pyatkov S. G. 2014. O nekotorykh klassicheskikh zadachakh dlya psevdoparabolicheskikh uravneniy [On some classes of inverse problems for pseudo-parabolic equations]. Matematicheskiye zametki SVFU, 21(2): 106-116.

10. Yuldashev T. K. 2016. Nelineynoe integro-differentsialnoe uravnenie psevdoparabolicheskogo tipa s nelokalnym integralnym usloviem [Nonlinear Integro-Differential Equation of Pseudoparabolic Type With Nonlocal Integral Condition]. Vestnik Volgogradskogo gosudarstvennogo universiteta. Seriya 1, Matematika. Fizika [Science Journal of Volgograd state university. Mathematics. Physics] 1(32): $11-23$.

11. Yuldashev T. K. 2017. Nelokal'naya krayevaya zadacha dlya neodnorodnogo psevdoparabolicheskogo integrirovaniya s differentsial'nym uravneniyem s vyrozhdennym yadrom [Nonlocal boundary value problem for a nonhomogeneous pseudoparabolic-type integro-differential equation with degenerate kernel] Vestnik Volgogradskogo gosudarstvennogo universiteta. Seriya 1, Matematika. Fizika [Science Journal of Volgograd state university. Mathematics. Physics], 1(38): 42-54.

12. Alikhanov A. A. 2015. A new difference scheme for the time fractional diffusion equation. Journal of Computational Physics, 280: 424-438.

13. Lyubanova A. Sh. 2017. The inverse problem for the nonlinear pseudoparabolic equation of filtration type, J. Sib. Fed. Univ. Math. Phys., 10(1): 4--15.

14. Gao G. H., Sun H. W., Sun Z. Z. 2015. Stability and convergence of finite difference schemes foraclass of time-fractional sub-diffusion equations based oncertain superconvergence. J. Comput. Phys., 280: 510-528.

15. Cui M. 2013. Convergence analysis of high-order compact alternating direction implicit schemes for the two-dimensional time fractional diffusion equation. Numer. Algorithms, 62: 383--409.

16. Gao G.H., Sun Z.Z., Zhang H. 2014. A new fractional numerical differentiation formula to approximate the Caputo fractional derivative and its applications, J.Comput. Phys. 259: 33--50.

17. Pang H.K., Sun H.W. 2012. Multigrid method for fractional diffusion equations, J. Comput. Phys., 231: $693--703$.

18. Calcagni G. 2012. Geometry of Fractional Spaces. Adv. Theor. Math. Phys., 16(2): 549--644.

19. Pimenov V. G., Hendy A. S. 2016. An implicit numerical method for the solution of the fractional advection-diffusion equation with delay. Trudy Inst. Mat. i Mekh. UrO RAN, 22(2): 218-226.

20. Pimenov V. G., Hendy A.S. 2016. Fractional analog of crank-nicholson method for the two sided space fractional partial equation with functional delay // Ural Math. J., 2(1): 48--57.

21. Pimenov V. G. 2018. Numerical methods for fractional advection-diffusion equation with heredity. J. Math. Sci. (N. Y.)., 230(5): 737-741.

Получена 28.05.2020

Бештоков Мурат Хамидбиевич - кандидат физико-математических наук, доцент, ведущий научный сотрудник Института прикладной математики и автоматизации КБНЦ РАН

ул. Шортанова, 89А, г. Нальчик, 360000, Россия

E-mail: beshtokov-murat@yandex.ru 